\title{
Ciclopirox Prodrug CPX-POM
}

National Cancer Institute

\section{Source}

National Cancer Institute. Ciclopirox Prodrug CPX-POM. NCI Thesaurus. Code C155886.

A phosphoryloxymethyl (POM) ester-based prodrug of ciclopirox (CPX), a synthetic, broad-spectrum antifungal agent with antibacterial, anti-inflammatory and potential antineoplastic activities. Upon intravenous administration of CPX-POM, the POM moiety is cleaved off by phosphatases and the active metabolite CPX is released. Although its exact anticancer mechanism is not yet fully elucidated, CPX has been shown to inhibit tumor cell proliferation, induce apoptosis, and reduce tumor cell mobility in certain cancer types. CPX inhibits Notch1 activation and inhibits the Notch1-mediated signaling pathway, which is upregulated in many cancer cell types. This inhibits Notch downstream target proteins, inhibits the expression of gamma-secretase complex proteins, and prevents proliferation in susceptible cancer cells. CPX inhibits the iron-containing enzymes, catalase and peroxidase, which facilitate the decomposition of hydrogen peroxide, a reactive oxygen species (ROS) involved in oxidative stress. CPX also inhibits the iron-dependent enzyme ribonucleotide reductase, which is essential in DNA synthesis. CPX downregulates protein expression of cyclin D1 and cyclin E1, as well as their enzymatic counterparts cyclin-dependent kinases 4 and 2 (CDK4 and CDK2), which may inhibit tumor cell proliferation by slowing cell cycle progression from G1/G0 to S phase. Further, CPX may induce apoptosis by downregulating the expression of anti-apoptotic proteins, $\mathrm{Bcl}-\mathrm{xL}$ and survivin, and increasing cleavage of $\mathrm{Bcl}-2$. Additionally, CPX may inhibit tumor cell proliferation, survival and motility by inhibiting the phosphorylation of p70 S6 kinase 1 (S6K1) and eukaryotic initiation factor 4E binding protein 1 (4E-BP1), two downstream effector molecules of the mammalian target of rapamycin complex 1 (mTORC1). The CPX-POM prodrug improves the solubility of CPX and increases systemic efficacy. 